\title{
Über die Gültigkeit des Lambert-Beerschen Gesetzes in Bromdampf
}

\author{
Von Gustav Kortüm und Dietrich Müller \\ Aus dem Physikal.-Chem. Institut der Universität Tübingen \\ (Z. Naturforschg. 1, 439-447 [1946]; eingegangen am 31. August 1945)
}

\begin{abstract}
Eine zusammenfassende Darstellung der bei der Prüfung des L a m b e r t - B e e r schen Gesetzes in Gasen auftretenden Fehlerquellen wird gegeben. Die bisher vorliegenden und zum Teil sich widersprechenden Arbeiten werden zusammengestellt und kritisch diskutiert. Eigene lichtelektrische Messungen bestätigen die Gültigkeit des L a m b e r t-B e e r schen Gesetzes bis zu Drucken von $100 \mathrm{~mm} \mathrm{Hg}$ und Temperaturen von etwa $600^{\circ} \mathrm{C}$ im Gebiet der sichtbaren Banden des Bromdampfes.
\end{abstract}

$I^{n}$ m Verlauf von Untersuchungen über den Einfluß zwischenmolekularer Kräfte auf das Absorptionsspektrum wurde auch die merkwürdige Zunahme der Absorption von Gasen bei Zusatz eines nicht absorbierenden Fremdgases näher untersucht. Dieser sog. „Fremdgaseffekt" ist in den sichtbaren Banden von Jod und Brom schon lange bekannt, doch widersprechen sich die früheren Angaben in manchen Punkten, so daß eigene Untersuchungen in Angriff genommen wurden. Wenn Zusatz eines fremden Gases die Absorption erhöht, sollte man a priori annehmen, daß auch bei Zusatz von Brom selbst die Absorption dieser jetzt unter einem höheren Druck stehenden Gesamtmenge größer ist als die Summe der Absorptionen der beiden Teilmengen. Die Absorption sollte also abhängig vom Druck und das Beersche Gesetz ungültig sein. In Vorversuchen ergaben sich gegen alle Erwartung keine merklichen Abweichungen, und wegen der Wichtigkeit dieser Frage zur Klärung des Fremdgaseffektes wurde eine möglichst exakte Prüfung des Beerschen Gesetzes unternommen.

Vorausgeschickt sei eine kurze Übersicht über die Absorptionsgebiete des Broms, dann eine Besprechung der Fehler, die bei den verschiedenen Untersuchungsmethoden auftreten können, und schließlich eine Zusammenstellung und kritische Diskussion der wesentlichen Arbeiten, in denen diese Frage bei den Halogenen untersucht wurde.

\section{Absorptionsgebiete}

Gasförmiges Brom besitzt im Sichtbaren starke nach Rot abschattierte Banden, die sich von
$6590 \AA$ an mit steigender Intensität nach Blau hin erstrecken und bei $5100 \AA$ A konvergieren. Ihre Rotationsstruktur ist sehr kompliziert, $P$ - und $R$-Zweig sind je dreifach, den drei Molekelsorten $\mathrm{Br}^{79,79}, \mathrm{Br}^{79,81}$ und $\mathrm{Br}^{81,81}$ (Häufigkeit $1: 2: 1$ ) entsprechend, ein $Q$-Zweig fehlt. Eine Schwingungs- und Rotationsanalyse gibt W.G.B row $\mathrm{n}^{1}$.

An die Konvergenzstelle schließt sich ein intensives Kontinuum an, das sein Maximum bei etwa $4150 \AA$ erreicht (vergl. Abb. 1). A. P. Acton, R. G. Aickin und N.S.Bayliss ${ }^{2}$ nahmen es bei verschiedenen Temperaturen auf und zeigten, daß es aus zwei Teilbanden zusammengesetzt ist; jedoch ist die Zuordnung $\mathrm{zu}$ den verschiedenen möglichen Elektronenübergängen ebenso wie bei den Bandensystemen noch nicht ganz gesichert, vor allem nicht, ob es sich hierbei um Interkombinationen vom Typ ${ }^{3} \Pi \leftarrow^{1} \Sigma$ (Singulett-Triplett) handelt, wie früher allgemein angenommen wurde (ausführliche Arbeiten hierzu bei O. Darbyshire ${ }^{3}$ und R.S. Mulliken ${ }^{4}$ ).

Ein sehr schwaches Bandensystem liegt im Rot zwischen 7600 und 6450 Å; möglicherweise schließt sich hieran ebenfalls nach kurzen Wellen zu ein allerdings schwaches Kontinuum an, das die Hauptbanden überlagert ${ }^{5,6}$. Z wischen 3400 und $2200 \AA$ liegt ein weiteres schwaches $\mathrm{Ab}$ sorptionsgebiet, das R. G. A i ckin und N.S.B ay -

1 Physic. Rev. [2] 38, 1179 [1931]; 39, 777 [1932].

2 J. chem. Physics 4, 474 [1936].

3 J. chem. Physics 4, 747 [1936].

4 J. chem. Physics 4, 620 [1936]; 8, 234 [1940]; Physic. Rev. [2] 57, 500 [1940].

5 W. G. B r o w n, Physic. Rev. [2] 37, 1007 [1930].

6 O. Darbyshire, Proc. Roy. Soc. [London] Ser. A 159, 93 [1937]. 
$\operatorname{liss}^{7}$ genauer untersuchten. Möglicherweise besitzt es Linienstruktur.

In Abb. 1 sind ferner die Absorptionskurven für flüssiges $\mathrm{Brom}^{8}$ und für die Lösung in $\mathrm{CCl}_{4}{ }^{9}$ aufgetragen. Die Absorption in den kondensierten Phasen liegt durchweg höher, für den Übergang von gasförmigem in gelöstes oder flüssiges Brom ist das Beer sche Gesetz nicht anwendbar. Die Ursache dafür liegt offenbar in den zwischenmolekularen Kräften, wenn auch die Gründe im einzelnen noch nicht genau bekannt sind; Betrachtungen hierüber siehe ${ }^{10}$ und ${ }^{11}$. Auffallend ist der große Unterschied unterhalb von etwa

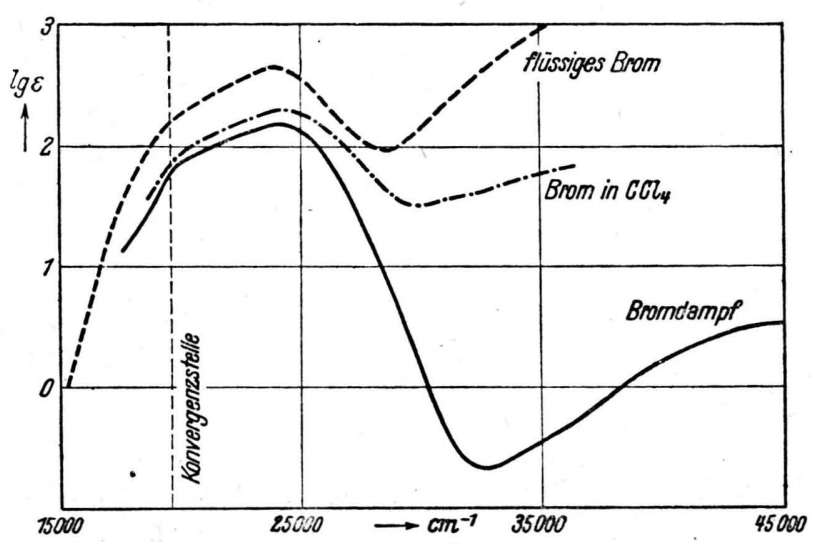

Abb. 1. Absorptionskurve von Brom.

$3300 \AA$; dieses Gebiet scheint also gegen Einwirkungen äußerer Kräfte sehr empfindlich zu sein. Aickin und Bayliss ${ }^{7}$ fanden hier auch einen sehr starken Fremdgaseffekt und Ungültigkeit des B eer schen Gesetzes schon im Gaszustand.

\section{Fehlerquellen}

Angesichts der oft widerspruchsvollen älteren Angaben erscheint es zweckmäßig, eine etwas ausführlichere Besprechung der Fehlerquellen vorauszuschicken. Vor allem muß dabei das B e e r sche Gesetz vom La mber tschen streng getrennt werden, eine Unterscheidung, die bisher nur in der Literatur, über ultrarote Spektren immer scharf genug durchgeführt wurde. Zwei völlig verschiedene Effekte können in einem Ban-

7 Trans. Faraday Soc. 34, 1371 [1938].

${ }^{8}$ D. Porret, Proc. Roy. Soc. [London] Ser. A 162, 414 [1937].

9 R. G. A ickin, N.S. B a y liss u. A. L. G. Rees, Proc. Roy. Soc. [London] Ser. A 169, 234 [1939]. denspektrum zu Täuschungen Anlaß geben: 1. die Erscheinung der „unvollständigen Absorption“ und 2. die Druckverbreiterung der Spektrallinien. Beide sind unabhängig voneinander und in ihren Wirkungen in gewissem Sinne entgegengesetzt.

1. „Unvollständige Absorption" und Lambertsches Gesetz bei Gasen.

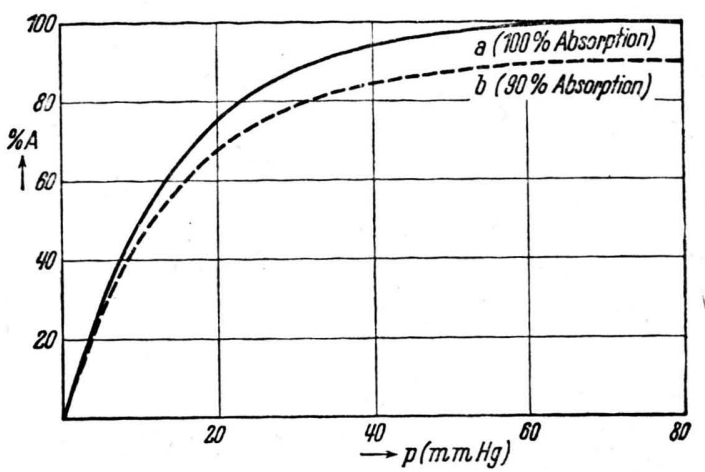

Abb. 2. Abhängigkeit der Absorption (in \%) von der Schichtdicke bei „unvollständiger Absorption“.

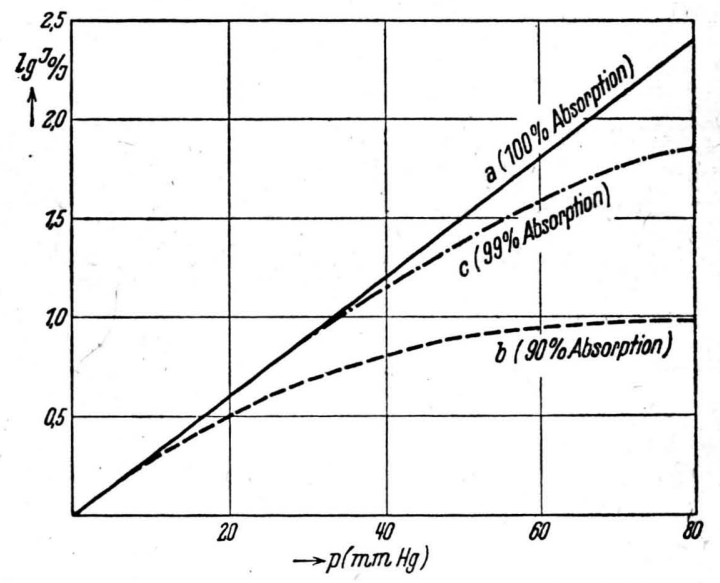

Abb. 3. Abhängigkeit der Extinktion von der Schichtdicke bei „,unvollständiger Absorption“.

Dieser Effekt läßt sich isolieren, indem man bei konstantem Druck arbeitet und nur die Schichtdicke variiert. Wenn der Absorptionskoeffizient in dem betrachteten Spektralbereich endlicher Breite konstant ist, wird das gesamte Licht in gleichen Schichten immer im gleichen Verhältnis geschwächt, wie.es das Lambertsche Gesetz verlangt. (Abweichungen hiervon wurden noch nicht beobachtet.) Die Absorption nähert sich also

10 C. L. Child u. O. J. Walker, Trans. Faraday Soc. 34, 1506 [1938].

11 N. S. B a y lis s u. A. L. G. Rees, J. chem. Physics 8,377 u. 492 [1940]. 
mit zunehmender Schichtdicke exponentiell dem Wert $100 \%$, und die Extinktion $\lg J_{0} / J$ steigt linear (vergl. Abb. 2 u. 3, Kurve $a$ ).

Völlig andere Verh̆ältnisse hat man in einem Bandenspektrum, in dem der Absorptionskoeffizient sich innerhalb des betrachteten Spektralbereichs sehr stark ändert. Er möge hierbei denselben Mittelwert haben wie vorher. Dann ist in den Linienmitten die Absorption wesentlich größer, während in den Zwischenräumen alles Licht ungehindert durchgeht. Durch Vergrößerúng der Schichtdicke ist in diesem Fall niemals eine vollständige Absorption zu erreichen. Der Absorptionskoeffizient wird von der Schichtdicke abhängig und damit das Lambertsche Gesetz scheinbar ungïltig. Kurve $b$ zeigt diesen Fall; dabei ist eine maximale Absorption von $90 \%$ angenommen. (Die Absorptionswerte entsprechen etwa unseren späteren Versuchsbedingungen, also $5461 \AA$ und $30 \mathrm{~cm}$ Schichtdicke bei Bromdampf.) Prinzipiell liegen hier dieselben Verhältnisse vor wie bei der Messung an steil abfallenden Ästen einer Lösungsbande ${ }^{12,13}$. Dieser Grenzwert der „unvollständigen Absorption“ hängt ab von dem

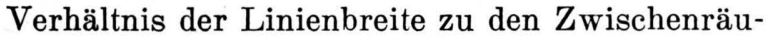
men ${ }^{14}$. Sind z. B. die Linien halb so breit, so liegt die maximale Absorption bei 33\%, vorausgesetzt, daß alle Linien gleich intensiv sind und kein kontinuierlicher Untergrund vorhanden ist. Die Schichtdicke, bei der sich diese Erscheinung zuerst bemerkbar macht, hängt von der Intensität der Linien ab; bei starker Absorption im Linienzentrum sollte der Effekt bereits bei relativ geringen Schichtdicken festzustellen sein.

Zuerst beobachtet wurde diese ,unvollständige Absorption" von Cl. Schaefer ${ }^{15}$ bei den Ultrarotbanden des $\mathrm{CO}_{2}$. Er schloß folgerichtig, daß die Banden Feinstruktur besitzen müßten, was erst einige Jahre später, bestätigt werden konnte. Es ist kein Zufall, daß diese Erscheinung in anderen Spektralgebieten in dieser Form bisher noch nicht beobachtet wurde; die Rotationsstruktur ist im Ultraroten sehr locker, im Gegensatz zu den meist äußerst dichten Strukturen der sichtbaren und ultravioletten Elektronenbanden. Prinzipiell ist „unvollständige Absorption“ und damit Ungültigkeit des L a m ber tschen Gesetzes in allen Bandenspektren $\mathrm{zu}$ erwarten, praktisch bemerkbar

12 G. K o r tü m, Kolorimetrie und Spektralphotometrie, Berlin 1942, S. 11 u. 32.

${ }_{13}$ G. Kortui m u. H. v. Halban, Z. physik. Chem. Abt. A 170, 212 [1934]. machen wird sie sich bei dichter Linienstruktur erst bei großen Schichtdicken. Zur Prüfung kann man statt der Schichtdicke auch die Intensität des einfallenden Lichtes ändern. In den Linienzentren bleibt das Intensitätsverhältnis des einfallenden zum durchgelassenen Licht konstant, dagegen addiert sich bei steigender Lichtintensität hierzu noch ein stetig wachsender Lichtstrom, der in den Zwischenräumen ungehindert durchgehen konnte. Das Intensitätsverhältnis von auffallendem zu durchgelassenem Licht und damit die Extinktion muß also abnehmen.

2. Druckverbreiterung der Spektrallinien und Lambertsches Gesetz.

Bei kleinen Drucken ( $p<1$ Atm.) ergibt sich nach der Lorentzschen Theorie der Stoßdämpfung eine Verbreiterung der Linie, die symmetrisch und dem Druck proportional ist. Da es sich um eine kurzzeitige Störung handelt, die im wesentlichen eine Phasenverschiebung der Strahlung hervorruft, wird die Gesamtintensität der Absorption nicht geändert. Man erhält also eine breitere Linie mit niedrigerem Maximum.

Bei höheren Drucken befindet sich die absorbierende Molekel dauernd im Störungsgebiet von Nachbarmolekeln. Die Druckverbreiterung läßt sich in diesem Fall statistisch berechnen; sie ist unsymmetrisch, d.h. es tritt eine Linienverschiebung auf ${ }^{16}$.

Die erste Beobachtung einer Druckverbreiterung bei den Halogenen machte R. W. W o od ${ }^{17}$. Um den Übergang vom kontinuierlichen Lösungszum diskreten Gasspektrum zu studieren, untersuchte er Jodlösungen in Schwefelkohlenstoff oberhalb der kritischen Temperatur und fand, daß bei geringer Jodkonzentration nur die kontinuierliche Lösungsbande vorhanden war; überstieg die Jodkonzentration einen gut reproduzierbaren Grenzwert, erschien auf dem kontinuierlichen Hintergrund das Bandensystem des Joddampfes. Merkwürdigerweise blieben dagegen selbst unter dem Druck von $250 \mathrm{Atm}$. Knallgas die Linien scharf und deutlich sichtbar, so daß er die Verwaschung nicht dem Druck zuschrieb, sondern eine Art Lösungszustand des Jods in gasförmigem $\mathrm{CS}_{2}$ und anderen organischen Lösungsmitteln annahm.

Direkte Messungen mit einem Stufengitter stell-

14 Th. Dreis ch, Handbuch d. Physik 19, 853.

15 Ann. Physik [4] 16, 93 [1905].

16 A. Unsöld, Vjschr. Astron. Ges. 78, 213 [1943].

17 Z. physik. Chem. 19, 689 [1896]. 
ten dann Chr. Füchtbauer ${ }^{18}$ sowie später M. G. Ribaud ${ }^{19}$, K. Vogt und J. Koenigsber ger ${ }^{20}$ und besonders eingehend I. I. A garbicean $u^{21}$ an. Übereinstimmend fanden sie die Halbwertsbreite bei Brom und Jod bei geringen Drucken zu etwa $0,035 \AA$; Steigerung des Eigendrucks sowie Zusatz von Fremdgasen erhöhte sie beträchtlich, wobei die Wirkung der verschiedenen Zusatzgase ziemlich gleich war. Als extremen Fall führen Vogt und Koenigsberger an, daß bei Zusatz von $20 \mathrm{Atm}$. $\mathrm{CO}_{2}$ zu Brom oder bei Erhitzen auf den gleichen Eigendruck die Linienstruktur völlig verwaschen wird. Nach F üchtbauer ${ }^{18}$ sinkt bei der Verbreiterung das Maximum der Linie, jedoch wurde noch nie exakt durch direkte Ausmessung der Linien bei den Halogenen geprüft, ob die Gesamtabsorption der Linie tatsächlich gleich bleibt. Eine Druckverschiebung der Bromlinien wurde ebenfalls beobachtet ${ }^{22,23}$; sie ist sehr gering und wird zu etwa $0,028 \AA$ pro Atm. angegeben.

Infolge der Druckverbreiterung wird die Linienstruktur mehr und mehr verwischt. Das wirkt sich nun auch auf die Erscheinung der ,unvollständigen Absorption“ in der Weise aus, daß die Abweichungen der Extinktion vom geradlinigen Verlauf sich erst bei größeren Schichtdicken bemerkbar machen. Außerdem wird dabei der Grenzwert der Absorption erhöht. Betrachten wir dazu den Fall, daß in den Linienzentren praktisch alles absorbiert wird, wir also den Grenzwert der Absorption schon erreicht haben. Dann läßt eine Verbreiterung der Linien die Absorption in den Linienflügeln stark ansteigen, während die Erniedrigung des Maximums kaum in Erscheinung tritt; es resultiert also eine Zunahme der Extinktion. Für den Grenzfall einer völligen Verwaschung der Linien wird offenbar die maximale $\mathrm{Ab}$ sorption gleich $100 \%$, Kurve $b$ geht also in Kurve $a$ über und das L a m bert sche Gesetz wird wieder gültig. Für geringere Drucke ergeben sich Kurven, die zwischen $a$ und $b$ liegen.

Auch diesen Effekt hat Schaefer ${ }^{15}$ beim $\mathrm{CO}_{2}$ beobachtet und diskutiert. Während durch Vergrößerung der Schichtdicke allein die Absorption niemals über einen bestimmten maximalen Wert

18 Physik. Z. 12, 722 [1911]

19 Ann. Physique [9] 12, 107 [1919].

20 Z. Physik 13, 292 [1923].

21 Ann. Physique [11] 2, 347 [1934].

22 A. Du four, C. r. hebd. Séances Acad. Sci. 145, 173 u. 757 [1907]. stieg, ließ sie sich durch Erhöhung des Druckes weiter steigern. Allerdings konnte er den Druck nicht so weit treiben, daß völlige Absorption erzielt wurde, doch war damit einwandfrei bewiesen, daß es sich um eine echte, ,unvollständige Absorption" und nicht um Streulicht handelte.

Eingehendere Betrachtungen über die Intensitätsverhältnisse bei Linienabsorption verdanken wir R.Ladenburg und F. Reiche ${ }^{24}$. Als Grenzgesetz für den Fall sehr dicker Schichten fanden sie unter Annahme einer Dispersionsverteilung der Linien für die Gesamtabsorption $A=$ const $\sqrt{N d i \gamma}$, wo $N=$ Zahl der Molekeln, $d=$ Dichte $f=$ Oszillatorenstärke und $\gamma=$ Dämpfungskonstante. Eine sehr gute Diskussion gibt auch W.Lochte-Holtgreven ${ }^{25}$.

Die Prüfung des Beerschen Gesetzes

Beide Fehlerquellen, „unvollständige Absorption" und Druckverbreiterung der Linien, werden bei der Prüfung des Beerschen Gesetzes in Erscheinung treten können. In dem Bereich, in dem das Lambertsche Gesetz gültig ist, also auf dem geradlinigen Teil der Extinktionskurve, sind auch keine scheinbaren Abweichungen vom Beerschen Gesetz zu erwarten, die durch die Linienstruktur bedingt wären, eine einwandfreie Prüfung ist also leicht möglich. In dem Bereich, in dem das La mbertsche Gesetz nicht mehr gilt, müssen natürlich auch scheinbare Abweichungen vom Beerschen Gesetz auftreten, selbst wenn dieses streng gültig wäre. Um es zu prüfen, muß man das Produkt $c \cdot d$ konstant halten, so daß man stets die gleiche Anzahl Molekeln unter verschiedenen Drucken beobachtet. Wahre Abweichungen der Extinktion machen sich dann in einer Druckabhängigkeit der Extinktion bemerkbar; sie beruhen auf der Wirksamkeit zwischenmolekularer Kräfte im weitesten Sinne, deren Einfluß bis zur Entstehung definierter Assoziationsprodukte gehen kann.

Bei Benutzung photographischer Methoden kann u.U. der Einfluß beider Fehlerquellen auf die Prüfung des B e er schen Gesetzes verschwinden, nämlich dann, wenn die Dispersion so groß ist, daß alle Linien in ihrer wahren Gestalt und Größe wiedergegeben werden, ohne sich zu überlappen. Jedoch zeigen Untersuchungen mit einer

23 A. P e r ot u. M. C o 11 i n e t, C. r. hebd. Séances Acad. Sci. 180, 2030 [1925].

24 Ann. Phvsik [4† 42. 181 [1913].

25 Z. Physik 103, 395 [1936]. 
Lummer-Gehrcke-Platte ${ }^{26}$, daß bei der außerordentlichen Schmalheit der Halogenlinien selbst bei höchster Dispersion die Intensitätsverteilung der Linien verzerrt wird. Als Fehler der Methode werden 15-20\% angegeben, eine exakte Prüfung ist also durch Messungen an den einzelnen Linien nicht möglich.

Daß beide Effekte auch bei der Intensitätsmessung einer einzelnen Spektrallinie beträchtliche Fehler bewirken können, haben Untersuchungen an Prädissoziationsspektren ${ }^{27}{ }^{28}$ gezeigt. Die Dispersion reichte nicht aus, um die Linien in ihrer wahren Schärfe wiederzugeben, so daß erst beträchtlich hinter der Prädissoziationsgrenze das Diffuswerden der Linien merklich wurde. Merkwürdigerweise trat jedoch genau an der Grenze, die durch Untersuchungen in Emission festgelegt werden konnte, eine beträchtliche Intensitätssteigerung der Linien auf. Da es sich um einen einheitlich verlaufenden Rotationszweig handelte, in dem derartige Intensitätsanomalien nicht $\mathrm{zu}$ erwarten waren, wurde eine analoge Deutung gegeben: die Prädissoziation bewirkt eine starke Verbreiterung der Linie bei gleichbleibender Gesamtabsorption, also ein Absinken des Maximums. In der Linienmitte wird mit und ohne Prädissoziation alles Licht absorbiert, dagegen steigt in den Linienflügeln die Absorption stark an, so daß insgesamt eine beträchtliche Intensitätssteigerung resultiert.

Bei allen anderen Verfahren wird dagegen über einen größeren Spektralbereich summiert, unabhängig davon, ob man visuell, photographisch mit geringer Dispersion oder lichtelektrisch mißt, ob man als Lichtquelle einen Ausschnitt aus einem Kontinuum oder eine einzelne Spektrallinie benutzt. Für die Brauchbarkeit der verschiedenen Methoden sind im wesentlichen dieselben Gesichtspunkte maßgebend wie bei der Untersuchung kontinuierlicher Spektren ${ }^{12}$. Allerdings erreicht die photographische Methode infolge der feinen Linienstruktur nur sehr geringe Genauigkeit; selbst im einfachsten Fall, dem Vergleich zweier gleicher Spektren, muß eine beträchtliche Unsicherheit in Kauf genommen werden. Am mei-

\footnotetext{
26 W. Opechowski, Acta physic. polon. 3, 307 [1934].

27 G. Herzber g, Molekülspektren, Dresden 1939, S. 299.

${ }_{28}$ L. F arkas, Z. Physik 70, 733 [1931].

28 ,Das Ultrarote Spektrum“, Berlin 1930.

30 G. B. B. M. Sutherland u. G. B. Callendar, Rep. Progr. Physics 9, 18 [1943].
}

sten empfiehlt es sich deshalb, als Lichtquelle eine kräftige Spektrallinie herauszufiltern und lichtelektrisch zu messen.

\section{Frühere Arbeiten}

Ausgedehnte Untersuchungen über die Gültigkeit des B e e r schen Gesetzes in Gasen liegen vor im ultraroten Gebiet. Hier stellte Ångström 1901 bei $\mathrm{CO}_{2}$ starke Abweichungen fest, und 1905 entdeckte Schaefer die schon besprochene „unvollständige Absorption". Im Verlauf weiterer Arbeiten wurde dann auch eine Erhöhung der Absorption durch Zusatz von Fremdgasen gefunden und vor allem durch v. Bahr und G. Hertz näher untersucht. Eine Zusammenfassung bis 1930 geben Cl. Schaefer und F. Matos si ${ }^{29}$, an neueren Arbeiten siehe ${ }^{30}$ und ${ }^{31}$. Es handelt sich hierbei immer nur um scheinbare Abweichungen, die durch die Linienstruktur hervorgerufen werden. Selbst beim Übergang in den flüssigen Aggregatzustand treten im allgemeinen nur geringe Abweichungen in den Banden auf ${ }^{32}$.

Im sichtbaren Gebiet hat D. L. Webster ${ }^{33}$ wohl als erster eine genauere Prüfung an den Halogenen vorgenommen (die Messungen von B. Goer ing ${ }^{34}$ sind völlig unzulänglich). Er benutzte ein Rohr mit mehreren hintereinanderliegenden Kammern, in die das Gas expandiert werden konnte, und verglich das erhaltene Spektrum visuell mit dem eines Vergleichsrohres. Uber den gesamten Absorptionsbereich des Chlors und im Kontinuum des Broms fand er das B e e r sche Gesetz für ein Druckverhältnis von 1:39 gut bestätigt, dagegen ergaben sich im Bandengebiet beträchtliche Abweichungen. Für ein Korttinuum ist die Versuchsanordnung einwandfrei, wenn auch die angegebene Fehlergrenze von $1 / \mathbf{2} \%$ zu niedrig sein dürfte. Der Vergleich eines Linienspektrums mit einem Vergleichsspektrum ist jedoch unzuverlässig, so daß die beobachteten Abweichungen damit wohl ihre Erklärung finden.

Umfangreiche sorgfältige Messungen über die $\mathrm{Ab}$ sorptionskurve, die Gültigkeit des Beerschen Gesetzes und den Fremdgaseffekt bei Brom verdanken wir M. G. Ribaud ${ }^{35}$. Seine Versuchsanordnung ist ähnlich wie bei We bste r ; im Kontinuum findet er ebenfalls das B e er sche Gesetz bis $150 \mathrm{~mm}$ Druck bestätigt. Außerdem beobachtet er mit hoher Dispersion das Verhalten der einzelnen Bromlinien bei Änderung von Druck, Temperatur und Zusatz von Fremdgasen.

In einem kurzen Auszug aus der unveröffentlichten Dissertation von G. D lu g o s ch ${ }^{36}$ wird das B e er sche Gesetz mit einem Spektralphotometer bei den HgLinien $5769 / 90,5461$ und $4916 / 60 \AA$ bei Jod bestätigt.

31 M. Summerfield u. J. Strong, Physic. Rev. [2] 60, 162 [1942].

${ }_{32}$ R. Mecke u. O. Vierling, Z. Physik 96, 559 [1935].

33 Physic. Rev. [2] 4, 177 [1914].

34 Dissertat. Königsberg 1907.

35 Ann. Physique [9] 12, 107 [1919].

36 Dissertat. Breslau 1923, Auszug. 
Weitere Messungen veröffentlichten K. Vogt und J.Koenigsberger ${ }^{20}$. Sie benutzten ein 6-m-Gitter und verglichen visuell bei konstantem $c \cdot d$ die Spektren von zwei Absorptionströgen. Bei Gültigkeit des Beerschen Gesetzes müssen beide Spektren in ihrer Helligkeit identisch sein; sie konnten dies sowohl im Bandengebiet als auch im Kontinuum bei Brom bis etwa $100 \mathrm{~mm}$, bei Jod bis 2,75 mm Druck bestätigen; darüber hinaus traten Abweichungen auf.

H. v. Halban und K. Siedent op $\mathrm{f}^{37}$ untersuchten die Absorption von Chlor mit ihrer lichtelektrischen Zweizellenmethode und fanden das B e er sche Gesetz bis zu 1 Atm. Druck innerhalb 0,5\% erfüllt.

Bei kinetischen Untersuchungen wurde öfter die Änderung der Bromkonzentration spektralphotometrisch verfolgt. Da hierbei jedoch mit breiten, herausgefilterten Lichtbändern gearbeitet wurde, ergaben sich bei den Eichmessungen naturgemäß mehr oder weniger große Abweichungen vom Beerschen Gesetz, die auf die mangelnde Spektralreinheit des Lichts zurückzuführen sind. W. Jost ${ }^{38}$ maß bei $5910 \AA$ A als Filterschwerpunkt, L. Pu s ch ${ }^{39}$ bei $4760 \AA$ (also noch im Kontinuum).

Im Zusammenhang mit dem Fremdgaseffekt untersuchten. V. Kondratjew und L. Polak photo. graphisch das Bandengebiet des Jods. Sie arbeiten mit konstantem Produkt $c \cdot d$ bei einem Schichtdickenverhältnis von 1:50 und fanden das Beersche Gesetz für Wellenlängen < $5430 \AA$ nicht erfüllt; gleichzeitig beobachteten sie hier einen starken Fremdgaseffekt. Dagegen sollte es oberhalb 5430 A gültig sein und kein Fremdgaseffekt auftreten. Dieses merkwürdige Ergebnis konnte allerdings von keinem anderen Beobachter bestätigt werden.

Mit einer völlig anderen Methodik gingen F. K o éh le ${ }^{41}$ und J.Harding ${ }^{42}$ an die Frage heran. Sie benutzten Quecksilberlinien als Lichtquelle und maßen die Absorption lichtelektrisch. Das Beersche Gesetz konnten sie bei Jod bestätigen, wenn auch $\mathrm{K}$ o e h le r eigenartigerweise nicht den geringsten Fremdgaseffekt fand, so daß seine Arbeit nicht ganz zuverlässig erscheint.

Bei Messungen der Absorptionskurve des Jods mit Monochromator und Photozelle fanden E. Rabinowitsch und W. C. W o od ${ }^{43}$ das Beer sche Gesetz im Kontinuum von Jod und Brom bestätigt.

Ưber das ultraviolette Kontinuum des Broms bei 3000 A liegen Untersuchungen vor von Aickin und Bayliss ${ }^{7}$. Das Beersche Gesetz erwies sich als ungültig, der Fremdgaseffekt war auffallend groß, und außerdem ergab sich für flüssiges Brom eine bis hundertfach stärkere Absorption als für gasförmiges. Da es sich hierbei jedoch vielleicht auch um ein sehr feines Bandensystem handelt. können die Abweichungen teilweise auch durch Ungültigkeit des Lambertschen Gesetzes bewirkt sein. Untersuchungen in dieser Richtung liegen noch nicht vor.

37 Z. physik. Chem. 103, 71 [1923].

38 Z. physik. Chem. Abt. B 3, 102 [1929].

${ }_{39}$ Z. Elektrochem. angew. physik. Chem. 24, 338 [1928].

40 Z. Physik 76, 386 [1932].
Neuerdings hat M. Kantze r ${ }^{44}$ die Gültigkeit des Beerschen Gesetzes bei Brom mit einem Gitter untersucht. Nach ihm soll der Absorptionskoeffizient im Bandengebiet mit steigendem Druck abnehmen (im Gegensațz zu Webster, Kondratjew und den Ultrarotarbeiten). Die Abweichungen sind von der Wellenlänge abhängig und können $60 \%$ erreichen.

Zum Schluß seien die früheren Untersuchungen in nebenstehender Tabelle zusammengestellt.

\section{Eigene Untersuchungen}

Methodik. Die genauesten und zuverlässigsten Ergebnisse sind bei lichtelektrischen Messungen zu erwarten, besonders bei Benutzung einer lichtstarken Spektrallinie. Im Fall des Broms empfiehlt sich die HgLinie $5461 \AA$ A. Sie liegt mitten im Bandengebiet, läßt. sich bequem mit Selenphotoelementen messen, ist sehr intensiv und vor allem relativ breit. Die Linie darf nämlich nicht vergleichbar schmal sein mit den Absorptionslinien, denn dann würde jede geringfügige Verwaschung oder gar spektrale Verschiebung beträchtliche scheinbare Änderungen der Absorption bewirken, weil dabei die gegenseitige Lage der Linien und der Grad ihrer Überschneidung sich ändern würden. Ein gutes Beispiel hierfür bieten die Linien 5769/90 ̊ einer sehr gut gekühlten Hg-Lampe bei geringer Belastung, die von Joddampf fast völlig durchgelassen werden; die entsprechenden Jodlinien liegen in den äußersten Linienflügeln der $\mathrm{Hg}$-Linien, so daß erst. bei. Verbreiterung der Linien durch stärkere Belastung der Hg-Lampe die Absorption merklich wird ${ }^{45}$.

Als Lichtquelle diente in unseren Versuchen eine Hg-Spektrallampe, die unter einem Betriebsdruck von etwa $3 \mathrm{Atm}$. brennt und deren grüne Linie, nach Messungen von B. R o m p e und R. S ch u l z ${ }^{46} \mathrm{zu}$ schließen, eine Breite von etwa 0,5-1 $\AA$ besitzt, während" die Breite der Bromlinien nur 0,035 $\AA$, also rund $1 / 20$ davon beträgt. Eine Reihe von Beobachtungen mit. höchster Dispersion ${ }^{\mathbf{1 8}, \mathbf{2 6}, 35}$ zeigt, daß je nach der benutzten Lampe 2-7 Bromlinien auf dem Grunde der Linie 5461 A liegen.

Die übrigen Hg-Linien wurden durch die SchottFilter $\mathrm{OG} 1+\mathrm{BG} 2 \mathrm{O}$ unterdrückt, jedoch zeigte es: sich, daß relativ beträchtliche Reste der Linien sowie das gesamte bei dieser Lampe ziemlich starke grüne Kontinuum durchgelassen wurden. Deshalb wurdenoch ein lichtstarker Spektrograph eingeschaltet (Steinheil GH, 3 Glasprismen, Helligkeit 1:3, Spalt $0,25 \mathrm{~mm}$ ), in dessen Bildebene eine Photozelle mit Spalt $(0,4 \mathrm{~mm})$ so angebracht war, daß gerade die Linie $5461 \AA$ a asgeblendet wurde. Lediglich die unmittelbar benachbarten Teile des Kontinuums lieBen sich auf diese Weise nicht entfernen, doch betrug ihre Intensität nach Vergleichsmessungen wesentlich weniger als $1 / 2 \%$ der Linie.

$$
\begin{aligned}
& 41 \text { Physic. Rev. [2] 44, } 761 \text { [1933]. } \\
& 42 \text { Philos. Mag. J. Sci. [7] 21, 773 [1936]. } \\
& 43 \text { Trans. Faraday Soc. 32, } 540 \text { [1936]. } \\
& 14 \text { C. r. hebd. Séances Acad. Sci. 214, } 998 \text { [1942]. } \\
& 45 \text { S. Mr o z ow s k i, Acta physic. polon. 3, } 447 \text { [1934]. } \\
& 46 \text { Z. Physik 108, } 654 \text { [1938]. }
\end{aligned}
$$




\begin{tabular}{|c|c|c|c|c|c|c|c|}
\hline 1 & Goering & 1907 & $\mathrm{Br}_{2}$ & & $\begin{array}{l}\text { visuell mit unge- } \\
\text { filtertem Licht }\end{array}$ & nein & (L a m b e rtsches Gesetz ungültig \\
\hline 2 & Webster & 1914 & $\begin{array}{l}\mathrm{Cl}_{2} \\
\mathrm{Br}_{2} \\
n\end{array}$ & $\begin{array}{l}\text { Kontin. } \\
\text { Banden }\end{array}$ & $\begin{array}{c}\text { spektroskopisch, } \\
\text { visuell mit Ver- } \\
\text { gleichsspektrum } \\
" \\
"\end{array}$ & $\underset{\text { nein }}{\mathrm{ja}}$ & $\varepsilon$ wächst mit steigendem Druck \\
\hline 3 & Ribaud & 1919 & $\begin{array}{l}\mathrm{Cl}_{2} \\
\mathrm{Br}_{2}\end{array}$ & Kontin. & & ja & bis $150 \mathrm{~mm}$ Druck \\
\hline 4 & Dlugosch & 1923 & $\mathrm{~J}_{2}$ & Banden & $\begin{array}{l}\text { Spektralphotome- } \\
\text { ter, Hg-Linien } \\
5769 / 90,5461 \\
4916 / 60 \AA\end{array}$ & ja & bis etwa $1 \mathrm{~mm}$ Druck \\
\hline 5 & $\begin{array}{l}\text { Vogt u. } \\
\text { Koenigsberger }\end{array}$ & 1923 & $\mathrm{Br}_{2}$ & $\begin{array}{l}\text { Kontin. } \\
\text { Banden }\end{array}$ & $\begin{array}{l}\text { visuell mit } \\
\text { Row land-Gitter }\end{array}$ & ja & $\begin{array}{l}\text { bis } 100 \text { mm Druck, darüber } \\
\text { Abweichungen }\end{array}$ \\
\hline & & & $\mathrm{J}_{2}$ & $\begin{array}{l}\text { Kontin. } \\
\text { Banden }\end{array}$ & $"$ & ja & $\begin{array}{l}\text { bis } 2,75 \mathrm{~mm} \text { Druck, darüber } \\
\text { Abweichungen }\end{array}$ \\
\hline 6 & $\begin{array}{l}\text { v. Halban u. } \\
\text { Siedentopf }\end{array}$ & 1923 & $\mathrm{Cl}_{2}$ & & $\begin{array}{l}\text { Lichtelektrische } \\
\text { Zweizellenmethode } \\
\text { Monochromator }\end{array}$ & ja & bis $1 \mathrm{Atm}$. Druck \\
\hline 7 & Pusch & 1928 & $\mathrm{Br}_{2}$ & Kontin. & $\begin{array}{l}\text { Spektralphotome- } \\
\text { ter, Filter } 4760 \AA\end{array}$ & nein & $\cdot$ \\
\hline 8 & Jost & 1929 & $\mathrm{Br}_{2}$ & $\begin{array}{l}\text { Banden } \\
\text { Kontin. }\end{array}$ & $"$ & nein & $\begin{array}{l}\varepsilon \text { fällt etwas mit steigendem } \\
\text { Druck }\end{array}$ \\
\hline 9 & $\begin{array}{l}\text { Kondratjew u. } \\
\text { Polak }\end{array}$ & 1932 & $\mathrm{Br}_{2}$ & Banden & $\begin{array}{l}\text { Photographisch, } \\
\text { kleines Gitter }\end{array}$ & $\underset{\text { nein }}{\mathrm{ja}}$ & $\begin{array}{l}\text { für } \lambda>5430 \AA \\
" \lambda<5430 \AA \\
\varepsilon \text { wächst mit steigendem Druck }\end{array}$ \\
\hline 10 & Koebler & 1933 & $\mathrm{~J}_{2}$ & Banden & $\begin{array}{l}\text { lichtelektr. Zwei- } \\
\text { zellenmethode, } \\
\text { Hg-Linien } \\
5769 / 90,5461 \AA\end{array}$ & ja & bis etwa $0,75 \mathrm{~mm}$ Druck \\
\hline 11 & Harding & 1936 & $\mathrm{~J}_{2}$ & $\begin{array}{l}\text { Banden } \\
\text { Kontin. }\end{array}$ & $\begin{array}{l}\text { lichtelektrisch, } \\
\mathrm{Hg} \text {-Linien } 5461 \mathrm{u} \text {. } \\
4358 \AA\end{array}$ & ja & bis $25 \mathrm{~mm}$ Druck \\
\hline 12 & $\begin{array}{l}\text { Rabinowitsch } \\
\text { u. Wood }\end{array}$ & 1936 & $\begin{array}{l}\mathrm{J}_{2} \\
\mathrm{Br}_{2}\end{array}$ & $\begin{array}{c}\text { Kontin. } \\
n\end{array}$ & $\begin{array}{l}\text { lichtelektrisch, } \\
\text { Monochromator } \\
\end{array}$ & $\begin{array}{l}\text { ja } \\
\text { ja }\end{array}$ & \\
\hline 13 & $\begin{array}{l}\text { Aickin u. } \\
\text { Bayliss }\end{array}$ & 1938 & $\mathrm{Br}_{2}$ & $\begin{array}{l}\text { Ultra- } \\
\text { violettes } \\
\text { Kontin. }\end{array}$ & photographisch & nein & $\varepsilon$ wächst mit steigendem Druck \\
\hline 14 & Kantzer & 1942 & $\mathrm{Br}_{2}$ & $\begin{array}{l}\text { Kontin. } \\
\text { Banden }\end{array}$ & $\begin{array}{l}\text { photographisch, } \\
\text { m.Rowland-Gitter }\end{array}$ & $\underset{\text { jein }}{\mathrm{ja}}$ & $\varepsilon$ nimmt ab mit steigendem Druck \\
\hline
\end{tabular}

Tab. Zusammenstellung der früheren Untersuchungen.

Bei dem Aufbau wurde vor allem sorgfältig darauf geachtet, ein schmales Bündel möglichst parallelen Lichts auszublenden, damit auch in den langen Absorptionsrohren keinerlei störende Reflexionen an den Wänden auftraten. Es wurde eine ZweizellenSubstitutionsmethode angewendet und durch eine unter $45^{\circ}$ geneigte Glasplatte ein Teil des Lichtbündels auf die Vergleichszelle geworfen. Die Haupt- menge durchlief das Absorptionsrohr und einen sehr exakten Graukeil, um dann von einer Kondensorlinse auf den Spektrographenspalt konzentriert zu werden. Als Nullinstrument diente ein Multiflexgalvanometer Typ MG 4. Die Kompensation war so gut, daß bei Schwankungen der Lampenstromstärke um 0,1 Amp. das Galvanometer völlig in Ruhe blieb.

Die Absorptionsrohre von 1, 12,5 und $30 \mathrm{~cm}$ Schicht- 
dicke waren aus Quarz mit aufgeschmolzenen Platten; durch ein Übergangsstück konnten sie mit einer Füllapparatur verbunden und bequem abgeschmolzen werden. Die Apparatur war auch zur Untersuchung des Fremdgaseffektes vorgesehen, sie enthielt ein Vorratsgefäß mit flüssigem Brom, ein Quecksilberund ein Schwefelsäuremanometer sowie einen Vorratskolben für Fremdgase. Das Brom war gewaschen, über $\mathrm{P}_{2} \mathrm{O}_{5}$ getrocknet und destilliert. Vor jeder Meßreihe wurde unter wiederholtem Ausfrieren evakuiert, längere Zeit Brom in die Apparatur gelassen, um eine oberflächliche Absättigung des Schliffettes zu erzielen, und schließlich wieder evakuiert. Das Quarzrohr war vorher an der Pumpe auf etwa $6000^{0}$ erhitzt, um Wasserspuren möglichst zu entfernen (es hatte sich gezeigt, daß sonst gelegentlich ein schwacher Belag auftrat, der die Ergebnisse fälschte).

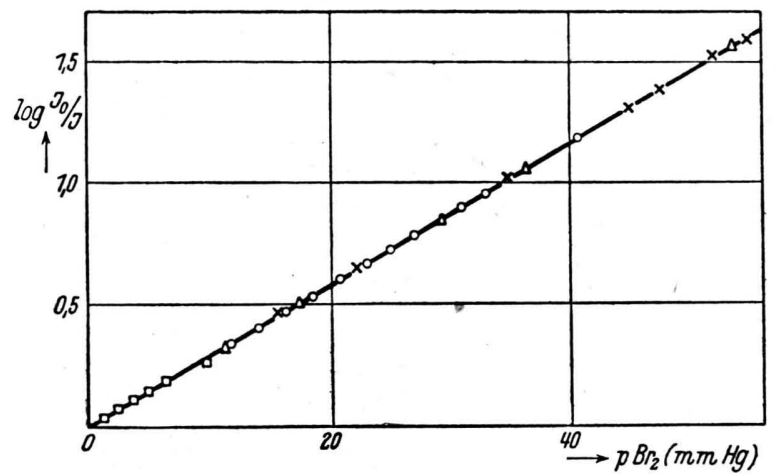

Abb.4. Prüfung des Beerschen Gesetzes bei Bromdampf. $\lambda=5461 \AA$, $d=30 \mathrm{~cm}$. (Die verschiedenen Zeichen entsprechen verschiedenen Meßreihen.)

Bei der Messung löste sich das Brom langsam in der Schwefelsäure des Manometers. Sowohl der Nullpunkt als auch der Umrechnungsfaktor in $\mathrm{mm} \mathrm{Hg}$ änderte sich dabei, so daß die Manometer häufig verglichen werden mußten. Bei der Korrektur blieb eine Unsicherheit von $1-2 \%$. Die verwendeten Drucke lagen so weit unterhalb des Sättigungsdruckes (etwa $150 \mathrm{~mm}$ bei Zimmertemperatur), daß man eine hinreichende Proportionalität zwischen Druck und Konzentration annehmen konnte. Es zeigte sich jedoch, daß die Fehler der Druckmessung bei weitem die Unsicherheit der Extinktionsbestimmung überstiegen. Um die hohe Genauigkeit der lichtelektrischen Methode besser ausnutzen zu können, machten wir außerdem eine Reihe von Messungen in abgeschmolzenen Rohren von 30 und $12,5 \mathrm{~cm}$ Schichtdicke bei konstantem Produkt $c \cdot d$. Dabei wurde die Konzentration durch Titration mit $n / 50$-Thiosulfat und Auswägen der Rohre mit Wasser bestimmt.

Weiter wurden photographische Aufnahmen bei konstantem $c \cdot d$ mit dem kleinen Gitterspektrographen von $\mathrm{Zei} ß$ gemacht. Bei der geringen Dispersion blieb die Feinstruktur der Banden völlig unaufgelöst. Zur Messung wurden die Rohre zunächst an der lichtelektrischen Apparatur mit Bromdampf auf gleiche Extinktion gefüllt, abgeschmolzen und dann mit einer
Punktlichtlampe als Lichtquelle eine Reihe von Doppelspektren je gleicher Belichtungszeit aufgenommen. Dabei wurde wieder sorgfältig darauf geachtet, daß ein enges Bündel paralleler Lichtstrahlen ausgeblendet wurde, um störende Wandreflexe zu vermeiden. Die Aufnahmen wurden mit einem Z e i $ß$-Spektrallinienphotometer ausgemessen.

\section{Ergebnisse}

Zunächst prüften wir, ob sich bei der verwendeten maximalen Schichtdicke von $30 \mathrm{~cm}$ schon eine „unvollständige Absorption“ bemerkbar macht, wie Goering ${ }^{34}$ behauptet, mit anderen Worten, ob das Lambertsche Gesetz noch gültig ist. Hierzu wurde die Extinktion des mit Bromdampf von etwa $24 \mathrm{~mm}$ Druck gefüllten 30-cm-Rohres bei verschiedenen Lichtintensitäten, die im Verhältnis $1: 2,4$ variierten, gemessen. Da die Charakteristik der Photoelemente nicht völlig identisch war, bei Schwächung des Lichts sich also der Nullpunkt verschob, wurde die Nullstellung bei jeder Messung neu bestimmt. Die sich so ergebenden Extinktionen unterschieden sich im Mittel bei drei verschiedenen Meßreihen um $-0,2 \%,+0,1 \%$ und $-0,6 \%$; selbst bei einer Lichtschwächung von 1:10 betrugen die $\mathrm{Ab}$ weichungen erst $+1,6 \%$, lagen also auch bei dieser wegen der geringen Lichtintensität ungenauen Messung durchaus noch innerhalb der Fehlergrenze. Das Lambertsche Gesetz erwies sich also als genügend erfüllt. Daraus geht hervor, daß die Messungen innerhalb der Genauigkeit der Methode (ca. 0,5\%) noch im Bereich der Kurve $\boldsymbol{a}$ (Abb. 2 bzw. 3) liegen, daß also die ,unvollständige Absorption" beim Bromdampf nur eine äußerst geringe Rolle spielen kann.

Das Beersche Gesetz wurde infolgedessen zunächst bei konstanter Schichtdicke und variablem Druck untersucht. Von den zahlreichen Meßreihen mit dem 30-cm-Rohr sind einige in Abb. 4 -wiedergegeben. Ihre Streuung dürfte infolge der Ungenauigkeit der Druckmessung etwa $\pm 1 \%$ betragen. Dabei ist noch zu bemerken, daß bei den Meßreihen, die sich bis zu Extinktionen über 1 erstreckten, die Anfangsintensität bei leerem Rohr nicht direkt gemessen werden konnte; sie ließ sich durch Extrapolation der sehr gut linear verlaufenden $\lg J$-Werte auf $p=0$ recht genau bestimmen. Mit $30 \mathrm{~cm}$ Schichtdicke wurde bis etwa $60 \mathrm{~mm}$, mit $1 \mathrm{~cm}$ bis $100 \mathrm{~mm}$ gemessen, doch waren die Fehler hierbei etwas größer, etwa $\pm 2 \%$. Der 
Grund dafür dürfte darin liegen, daß bei diesen relativ nahe an der Sättigungsgrenze liegenden Drucken das ideale Gasgesetz nicht mehr so gut erfüllt ist. Auch bei höheren Temperaturen bis zu $600^{\circ} \mathrm{C}$ erwies sich das Beersche Gesetz innerhalb der angegebenen Grenzen als gültig.

Einige Messungen wurden bei Zimmertemperatur mit abgeschmolzenen Rohren und konstantem Produkt $c \cdot d$ gemacht. Die Konzentration wurde durch Titration bestimmt, die Genauigkeit lag also erheblich höher. Bei 4 Bestimmungen betrugen die Fehler $+0,42 \%,+0,29 \%,-0,62 \%$ und $+0,75 \%$ bei einer Extinktion von etwa 0,6 .

Es ist auffallend, daß gerade alle Autoren, die lichtelektrisch arbeiteten, das Beersche Gesetz bestätigt fanden. Für die lichtelektrische Methode spricht zunächst ihre bei relativen Messungen leicht erreichbare große Genauigkeit. Als prinzipielle Fehlerquelle käme bei der hier angewendeten Methode nur in Frage, daß durch eine Verbreiterung oder Verschiebung der Bromlinien gegenüber der Hg-Linie sich die Absorption ändert - und diese Änderung müßte dann gerade die (wahren) Abweichungen vom Beerschen Gesetz innerhalb weniger als 1\% kompensieren. Das erscheint um so unwahrscheinlicher, als diese Kompensation dann in dem weiten Temperaturintervall von 20 bis $600^{\circ} \mathrm{C}$ stattfinden müßte. Zur eindeutigen Widerlegung dieser Möglichkeit wurde auch mit einem kontinuierlichen Lichtbündel von etwa $100 \AA$ Breite bei verschiedenen Wellenlängen zwischen 6000 und $5000 \AA$ gemessen. Dabei waren natürlich größere Fehler zu erwarten, immerhin stiegen sie nicht über $\pm 2-3 \%$.

Um beurteilen zu können, wie weit die photographische Methode bei Bandenspektren anwendbar ist, wurde eine Reihe von Aufnahmen bei konstantem Produkt $c \cdot d$ gemacht, nachdem die Extinktion lichtelektrisch genau bestimmt war. Schon rein visuell fielen kleine Unterschiede im Gesamtbild des Bandensystems auf. Bei der Ausphotometrierung stimmten die Schwärzungswerte im Kontinuum auf einige Prozente überein, im Bandengebiet jedoch streuten die Werte sehr stark und unregelmäßig. Bei der geringen Auflösung überlappen sich die Linien, bei einer Verbreiterung kann sich das Gesamtbild wesentlich ändern, wie auch bei Aufnahmen mit etwas höherer Dispersion wiederholt festgestellt wurde.

Eine weitere Fehlerquelle bei photographischen Intensitätsmessungen an unaufgelösten Bandensystemen könnte in folgendem zu suchen sein: In der Nähe der Linienzentren ist die Absorption so stark, daß der Schwellenwert der Platte nicht immer erreicht wird. Bei der Mittelung über mehrere Linien wird deshalb die Schwärzung der Platte zu gering und damit die Absorption zu hoch erscheinen. Durch die Verbreiterung der Linien wird die Absorption sich mehr dem wahren Wert nähern, also etwas abnehmen. Versuche, diesen Effekt genauer zu fassen, schlugen fehl, da die Fehlerbreite der Aufnahmen zu groß war.

Hiernach dürfte die photographische Methode zur Prüfung des Beerschen Gesetzes bei Bandenspektren völlig ungeeignet sein. Wahre Abweichungen infolge der ziemlich schwachen gegenseitigen Wechselwirkungskräfte wären bei geringen Drucken höchstens in der Größenordnung von einigen Prozenten zu erwarten, sie würden sich also bei den Aufnahmen, deren Fehlergrenze man auf $\pm 10-20 \%$ schätzen kann, gar nicht bemerkbar machen.

Im Kontinuum konnten wir photographisch in Übereinstimmung mit den früheren Arbeiten die Gültigkeit des Beerschen Gesetzes bestätigen, ebenfalls lichtelektrisch mit der Hg-Linie $4360 \AA$ und einer Alkalizelle mit einer Genauigkeit von $\pm 2 \%$.

Nach unseren Messungen kann die Gültigkeit des Beerschen Gesetzes im Bandengebiet des Broms bei Drucken bis zu $100 \mathrm{~mm}$ und Temperaturen bis $\mathrm{zu} 600^{\circ} \mathrm{C}$ als gesichert gelten. Widersprechende Angaben dürften auf Verwendung ungeeigneter Methoden zurückzuführen sein. Dieses Ergebnis wird sich für die Deutung des eingangs: erwähnten „Fremdgaseffektes“ als wichtig erweisen, worüber demnächst berichtet werden soll.

Der Steinheil-Spektrograph wurde uns von Hrn. Prof. Butenandt für unsere Messungen in freundlichster Weise zur Verfügung gestellt, wofür wir unseren herzlichsten Dank aussprechen. 